Relations industrielles

Industrial Relations

\title{
Index - Volume $71-2016$
}

Volume 71, numéro 4, automne 2016

URI : https://id.erudit.org/iderudit/1038617ar

DOI : https://doi.org/10.7202/1038617ar

Aller au sommaire du numéro

\section{Éditeur(s)}

Département des relations industrielles de l’Université Laval

\section{ISSN}

0034-379X (imprimé)

1703-8138 (numérique)

Découvrir la revue

\section{Citer ce document}

(2016). Index - Volume $71-2016$. Relations industrielles / Industrial Relations, 71(4), 778-782. https://doi.org/10.7202/1038617ar

Tous droits réservés @ Département des relations industrielles de l’Université Laval, 2016
Ce document est protégé par la loi sur le droit d'auteur. L'utilisation des services d'Érudit (y compris la reproduction) est assujettie à sa politique d'utilisation que vous pouvez consulter en ligne.

https://apropos.erudit.org/fr/usagers/politique-dutilisation/ 


\section{Index - Volume $71-2016$}

\section{VOLUME 71-1}

\section{Articles}

Amélioration ou polarisation? Évolution de la structure et de la qualité des emplois au Québec et au Canada, 1997-2013

PAUL-ANDRÉ LAPOINTE ET CATHERINE BACH

Supply Chain Leverage and Regulating Health and Safety Management in Shipping.

DAVID WALTERS, PHILIP JAMES, HELEN SAMPSON, SYAMANTAK BHATTACHARYA,

CONGHUA XUE AND EMMA WADSWORTH

La pratique de la négociation basée sur les intérêts dans les processus

de négociation collective : perceptions et appropriations syndicales

MARC-ANTONIN HENNEBERT, MARCEL FAULKNER ET REYNALD BOURQUE

Understanding the Pathways to Above-Mandatory Severance Pay

When Downsizing: A Qualitative Comparative Analysis of 20 Cases in France

PIERRE GARAUDEL, RACHEL BEAUJOLIN, FLORENT NOËL AND GÉRALDINE SCHMIDT

L'interaction entre facteurs organisationnels et locus de contrôle

dans la prédiction de l'engagement organisationnel affectif

XAVIER PARENT-ROCHELEAU, GILLES SIMARD, KATHLEEN BENTEIN

ET MICHEL TREMBLAY

Varieties of Capitalism: A Critique.

TRAVIS WILLIAM FAST

Culture organisationnelle, condition de l'organisation du travail

et épuisement professionnel

JULIE DEXTRAS-GAUTHIER ET ALAIN MARCHAND

\section{VOLUME 71-2}

\section{Articles}

Understanding Turnover as a Lifecycle Process: The Case of Young Nurses. NANCY CÔTÉ

La diffusion de l'instrumentation de la gestion des compétences

en Amérique du Nord depuis David C. McClelland

DOMINIQUE BOUTEILLER ET PATRICK GILBERT

Words at Work: Constructing a Labour Conflict.

YONATAN RESHEF AND CHARLES KEIM

Le transfert des pratiques de flexibilisation de l'emploi dans une firme

multinationale : le rôle charnière des acteurs locaux des filiales. 
Three Worlds of Professional and Managerial Staff in France. JENS THOEMMES

Insatisfaction des salariés et des clients en réaction à l'automatisation des caisses d'un hypermarché : de la prise de parole à la défection et à la négligence AMADOU BA ET DAVID ALIS

Maximizing the Benefits of Internationalization: The Moderating Role of Labour Flexibility. 350

HEUNG-JUN JUNG, SUNG-CHUL NOH AND SUN-WOOK CHUNG

\section{VOLUME 71-3}

Tribute to Richard J. Long/Hommage à Richard J. Long

MARC MENTZER AND ALLEN PONAK

\section{Articles}

Quand le syndicat devient vecteur d'inégalités: les effets des clauses « orphelins» sur l'association syndicale

DIANE GAGNÉ ET MARIE-JOSÉE DUPUIS

Safeguarding Workers: A Study of Health and Safety Representatives

in the Queensland Coalmining Industry, 1990-2013

DAVID WALTERS, RICHARD JOHNSTONE, MICHAEL QUINLAN AND EMMA WADSWORTH

Pratiques de conciliation travail-famille et détresse psychologique des salariés québécois: une comparaison selon le genre.

MAUDE BOULET ET CÉLINE LE BOURDAIS

The Invisibilization and Denial of Work in Argentinian

Garment Homework.

ROSARIA BURCHIELLI AND ANNIE DELANEY

Analyse des composantes de la charge de travail perçue par les cadres dans un contexte de gestion des compétences

NARGES SASSI ET HAZEM BEN AISSA

The Effects of the Psychological Contract among Professional Employees

Working in Non-Professional Organizations

PASCAL PAILLÉ, NICOLAS RAINERI AND PATRICK VALEAU

L'identification au contenu du travail comme déterminant du souhait de rester dans un emploi. 


\section{VOLUME 71-4}

\section{Articles}

Multi-Scalar Trade Unionism : Lessons from Maritime Unions. 589 PETER FAIRBROTHER AND VICTOR OYARO GEKARA

Le système d'emploi des travailleurs agricoles saisonniers : topographie d'un rapport salarial multipartite

DALIA GESUALDI-FECTEAU

What's Next after Psychological Contract Violation?

SYLVIE GUERRERO AND MICKAEL NAULLEAU

Le rôle médiateur de l'engagement au travail dans la relation entre

la justice organisationnelle et les comportements de citoyenneté :

le cas de la police française.

MATHIEU MOLINES ET AMAR FALL

Analyzing Exclusion in Global Worker Health Policy.

JEFFREY HILGERT

Analyse des processus menant à des changements dans une entreprise du secteur

aéronautique : vers un modèle d'évaluation des interventions ergonomiques.

VALÉRIE ALBERT, NICOLE VÉZINA, HENRIETTE BILODEAU ET FABIEN COUTAREL

Labour Standards in Global Value Chains: Disentangling Workers' Voice, Vicarious Voice, Power Relations, and Regulation.

EMMANUEL JOSSERAND AND SARAH KAINE

\section{RECENSIONS / BOOK REVIEWS}

\section{VOLUME 71-1}

Perspectives multidimensionnelles sur les restructurations d'entreprise

Sous la direction de Patrice Jalette et Linda Rouleau

FRANÇOIS BOLDUC

Les agences de travail temporaire : leur rôle et leur fonctionnement comme intermédiaires du marché du travail

Sous la direction de Mircea Vultur et Jean Bernier

MARIE-LAURE DIOH

Prévenir les problèmes de santé mentale au travail:

contribution d'une recherche-action en milieu scolaire

Par Marie-France Maranda, Simon Viviers et Jean-Simon Deslauriers

MARIE-MICHELLE GOUIN

Sports and Labor in the United States

By Michael Schiavone

BRAHAM DABSCHECK 


\section{VOLUME 71-2}

Le Capital au XX|e siècle

Par Thomas Piketty

MARC LAVOIE

International and Comparative Employment Relations. National regulation,

global changes

By Greg J. Bamber, Russell D. Lansbury, Nick Wailes and Chris F. Wright

ANTHONY GOULD

Emploi, formation, compétences: les régulations de la relation salariale en questions

Sous la direction d'Elodie Béthoux, Jean-Vincent Koster, Sylvie Monchatre, Frédéric Rey, Michèle Tallard et Catherine Vincent

JEAN-LUC BÉDARD

Ressources humaines. Gérer les personnes et l'ordre social dans l'entreprise.

Par Évelyne Léonard

SYLVAIN LUC

L'intervention en santé et en sécurité du travail. Pour agir en prévention

dans les milieux de travail

Sous la direction de Sylvie Montreuil, Pierre-Sébastien Fournier et Geneviève Baril-Gingras

RENÉE FORTIN

\section{VOLUME 71-3}

Ten Pathways to Death and Disaster.

By Michael Quinlan

MICHEL PÉRUSSE

Les critiques de la gestion

Par Jean Nizet et François Pichault

FRANÇOIS BERNARD MALO

The Philosophical Foundations of Management Thought

By Jean-Etienne Joullié and Robert Spillane

ANTHONY M. GOULD

(R)évolution du management des ressources humaines.

Des compétences aux capabilités.

Coordonné par Solveig Fernagu Oudet et Christian Batal

JUSTINE ARNOUD ET CATHY KROHMER

Working through the Past: Labor and Authoritarian Legacies

in Comparative Perspective

Edited by Teri L. Caraway, Maria Lorena Cook and Stephen Crowley

TRAVIS WILLIAM FAST 
Empty Labor. Idleness and Wokplace Resistance...

Par Roland Paulsen

MIRCEA VULTUR

Responsabilité sociale des entreprises: mirage ou réalité ?.

Par Mustapha Bettache

CLAIRE DUPONT

Refonder le système de protection sociale.

Pour une nouvelle génération de droits sociaux.

Par Bernard Gazier, Bruno Palier et Hélène Périvier

YASMINE MOHAMED

\section{VOLUME 71-4}

Mentir au travail..

Par Duarte Rolo

FREDERICK PLAMONDON

Reflexive Labour Law in the World Society.

Ralf Rogowski

ME P. MARTIN DUMAS

Détroit pas d'accord pour crever. Une révolution urbaine.

Par Dan Georgakas et Marvin Surkin

SARI MADI

Building Global Labor Solidarity in a Time of Accelerating Globalization

Edited by Kim Scipes

BRAHAM DABSCHECK

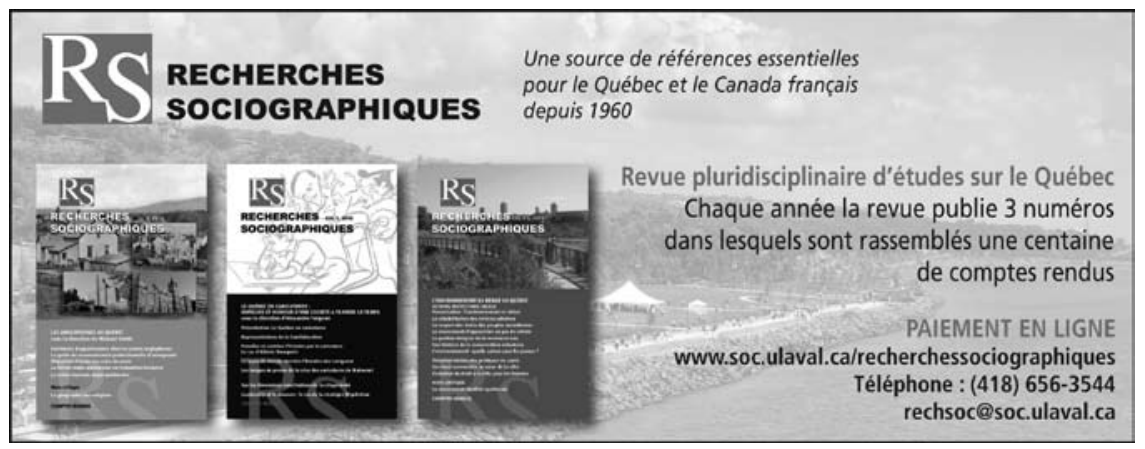

